\title{
Aphids (Hemiptera, Aphididae) on ornamental plants in greenhouses in Bulgaria
}

\author{
Mariya Yovkova', Olivera Petrović-Obradović ${ }^{2}$ Elena Tasheva-Terzieva ${ }^{3}$, \\ Aneliya Pencheva'
}

I Faculty of Ecology and Landscape Architecture, University of Forestry, 10 Kliment Ohridski Blvd, 1756 Sofia, Bulgaria 2 Faculty of Agriculture, University of Belgrade, 6 Nemanjina str., 11080 Beograd-Zemun, Serbia 3 Faculty of Biology, Sofia University "St. Kliment Ohridski", 8 Dragan Tsankov Blvd, 1164 Sofia, Bulgaria

Corresponding author: Mariya Yovkova (mariya_yovkova@abv.bg)

Academic editor: A. Popov | Received 15 November 2012 | Accepted 12 July 2013 | Published 30 July 2013

Citation: Yovkova M, Petrović-Obradović O, Tasheva-Terzieva E, Pencheva A (2013) Aphids (Hemiptera, Aphididae) on ornamental plants in greenhouses in Bulgaria. In: Popov A, Grozeva S, Simov N, Tasheva E (Eds) Advances in Hemipterology. ZooKeys 319: 347-361. doi: 10.3897/zookeys.319.4318

\begin{abstract}
Investigations on the species composition and host range of aphids on ornamental greenhouse plants in Bulgaria was conducted over a period of five years, from 2008 to 2012. Twenty greenhouses, growing ornamentals for landscaping, plant collections and other purposes were observed. They were located in the regions of Sofia, Plovdiv, Smolyan, Pavlikeni, Varna and Burgas. The total number of collected aphid samples was 279. Their composition included 33 aphid species and one subspecies from 13 genera and 5 subfamilies. Twenty-eight species were found to belong to subfamily Aphidinae. Almost $70 \%$ of all recorded species were polyphagous. The most widespread aphid species was Myzus persicae, detected in 13 greenhouses all year round, followed by Aulacorthum solani (10 greenhouses) and Aphis gossypii (9 greenhouses). The widest host range was shown by Myzus persicae (43 hosts), Aulacorthum solani (32 hosts) and Aulacorthum circumflexum (23 hosts).

The list of host plants includes 114 species from 95 genera and 58 families. The greatest variety of aphid species was detected on Hibiscus (9 species). Out of all aphid samples $12.9 \%$ were collected on Hibiscus and $6.8 \%$, on Dendranthema. The greatest variety of aphid species was detected on Hibiscus (9 species).
\end{abstract}

Copyright Mariya Yovkova et al. This is an open access article distributed under the terms of the Creative Commons Attribution License 3.0 (CC-BY), which permits unrestricted use, distribution, and reproduction in any medium, provided the original author and source are credited. 
Periphyllus californiensis and Aphis (Aphis) fabae mordvilkoi are reported for the first time for Bulgaria. Furthermore, Aphis spiraecola has been found in new localities and has widened its host range in this country.

\section{Keywords}

Aphididae, aphids, ornamental plants, greenhouses, Bulgaria

\section{Introduction}

Aphids cause serious damage in greenhouses, where conditions are favorable for their development throughout the year and where they can reach high density over a short period of time. The damage caused by aphids may lead to deterioration of the ornamental qualities of infested plants and sometimes even death. One of the most important and serious consequences is virus transmission.

Currently, there is no extensive research on ornamental plants in greenhouses in Bulgaria, which prompted the current study. The only survey on this topic was conducted by Tashev (1962). He reported 6 aphid species: Aulacorthum (Neomyzus) circumflexum (Buckton, 1876); Macrosiphoniella sanborni (Gillette, 1908); Macrosiphum euphorbiae (Thomas, 1878); Myzus (Myzus) ornatus Laing, 1932; Myzus (Nectarosiphon) ascalonicus Doncaster, 1946; Myzus (Nectarosiphon) persicae (Sulzer, 1776).

The results of our survey contribute to the scientific knowledge in the investigated field, but also have a practical application, benefitting producers of ornamental crops. The purpose of this study was to identify the species composition of aphids on greenhouse ornamentals, their host range and the most frequently infested ornamental species in Bulgaria.

\section{Material and methods}

The investigation was conducted over a period of five years, from 2008 to 2012. Twenty greenhouses, located in the regions of Sofia, Plovdiv, Smolyan, Pavlikeni, Varna and Burgas, were observed. Several types of greenhouses were included: for growing and propagation of ornamentals, for landscaping (annuals, perennials, rooting cuttings), for acclimatization of imported plants, for winter preservation of cold-tender species and for plant collections.

The observed greenhouses are designated as follows:

Greenhouses with a permanent plant composition: Greenhouses of University of Forestry (GL); Greenhouses of Bulgarian Academy of Science (GB); Greenhouses of the University Botanic Gardens (GS); Greenhouses of Euxinograd park (GE); Greenhouses of Vrana park (GV); Greenhouses of Krichim park (GK); Ravda (R1); 
Greenhouses with a constant circulation of plant species: private greenhouses in Sofia (S1, S2, S3, S4, S5, S6); Varna (V1, V2); Burgas (B1); Ravda (R2); Pavlikeni (PV); Plovdiv (PL); Smolyan (SM).

Aphids were collected in plastic bags together with the infested plant parts. Larvae were reared in laboratory conditions to the stage of adults.

The species identification was carried out using permanent microscope slides, after the traditional method of Hille Ris Lambers (1950). Identification keys included Blackman and Eastop (1994, 2000, 2006), Shaposhnikov (1964) and Stroyan (1984).

\section{Results}

The total number of collected samples of aphids on ornamental plants during the observed five-year period was 279 .

In total, 33 aphid species and one subspecies from 13 genera and 5 subfamilies (Lachninae, Chaitophorinae, Calaphidinae, Aphidinae and Eriosomatinae) were identified. Four species were identified only to the generic level but they are included in a total number of species because of the presence of clear distinguishing characters proving that they are separate species. Fourteen species belong to genus Aphis ( $42 \%$ ) and three species belong to genus Myzus (9\%). Genera Aulacorthum, Brachycaudus, Macrosiphum and Rhopalosiphum are represented by 2 species. Genera Cinara, Idiopterus, Macrosiphoniella, Ovatus, Periphyllus, Prociphilus and Tinocallis are represented by 1 species.

All aphid species and their host plants, including the data of Tashev (1962), are presented in alphabetical order in Table 1 .

\section{Discussion}

Twenty three of all recorded aphid species $(69.7 \%)$ have been reported on indoor ornamental plants (Weiss 1916, Cichocka and Goszczynski 1975, Achremowicz et al. 1986, Cichocka 1992, Łabanowski 2008, Rafi et al. 2010) and only six (18.2\%) have been found on ornamentals in Bulgarian greenhouses (Tashev 1962).

Twenty one of all recorded aphid species are polyphagous, 9 are oligophagous and only one is monophagous (Blackman and Eastop 1994, 2000, 2006). Seven of the polyphagous species were observed more frequently (Fig. 1).

During the survey, the most common species, represented by the highest numbers of samples, was Myzus persicae, found throughout the year in 13 greenhouses (61 samples, $21.9 \%$ ) (Fig. 1), followed by Aulacorthum solani (10 greenhouses, 51 samples, $18.3 \%$ ), Aulacorthum circumflexum (3 greenhouses, 33 samples, $11.8 \%$ ), Aphis gossypii (9 greenhouses, 26 samples, $9.3 \%$ ), Aphis spiraecola (4 greenhouses, 11 samples, $3.9 \%$ ), Macrosiphum euphorbiae (3 greenhouses, 9 samples, $3.2 \%$ ) and Aphis fabae 
Table I. Aphid species on greenhouse ornamental host plants recorded in Bulgaria.

\begin{tabular}{|c|c|c|c|c|}
\hline Aphid species & Host plant family & Host plant species & \begin{tabular}{|l|}
$\begin{array}{l}\text { Green- } \\
\text { house }\end{array}$ \\
\end{tabular} & Date \\
\hline \multirow[t]{5}{*}{ Aphis (Aphis) craccivora Koch, 1854} & \multirow[t]{2}{*}{ Fabaceae } & Robinia pseudoacacia L. & GL & 27.07 .2010 \\
\hline & & Wisteria chinensis Siebold & R1 & 17.07.2009 \\
\hline & Malvaceae & Hibiscus rosa-sinensis $\mathrm{L}$. & GL & 11.07 .2011 \\
\hline & Nyctaginaceae & Bougainvillea glabra Choisy & GE & 27.05 .2009 \\
\hline & Portulacaceae & Portulaca umbraticola Kunth & B1 & 17.08 .2010 \\
\hline \multirow[t]{7}{*}{ Aphis (Aphis) fabae Scopoli, 1763* } & Agavaceae & Yucca elephantipes Hort. ex Regel & S1 & 05.07 .2011 \\
\hline & \multirow[t]{2}{*}{ Aizoaceae } & \multirow[t]{2}{*}{ Aptenia cordifolia (L.f.) Schwantes } & \multirow[t]{2}{*}{ GL } & 18.09 .2009 \\
\hline & & & & 30.06 .2009 \\
\hline & Araceae & Anthurium andraeanum Linden & GE & 01.06 .2010 \\
\hline & Asteraceae & Cosmos bipinnatus Cav. & GL & 11.07 .2011 \\
\hline & Nyctaginaceae & Bougainvillea glabra Choisy & GE & 13.07.2009 \\
\hline & Solanaceae & Datura hybrida Ten. & GE & 27.05 .2009 \\
\hline $\begin{array}{l}\text { Aphis (Aphis) fabae mordvilkoi } \\
\text { Börner \& Janich, } 1922\end{array}$ & Tropaeolaceae & Tropaeolum majus L. & GE & 01.06 .2010 \\
\hline Aphis (Aphis) ex gr. fabae & Araceae & Anthurium andraeanum Linden & GE & 01.06 .2010 \\
\hline $\begin{array}{l}\text { Aphis (Aphis) farinosa Gmelin, } \\
1790\end{array}$ & Salicaceae & Salix matsudana Koidz. & GB & 26.05 .2010 \\
\hline Aphis (Aphis) ex gr. frangulae & Asteraceae & $\begin{array}{l}\text { Gazania heterophylla Willd. ex } \\
\text { Steud. }\end{array}$ & S2 & 06.08 .2010 \\
\hline \multirow[t]{26}{*}{ Aphis (Aphis) gossypii Glover, 1877} & \multirow[t]{4}{*}{ Acanthaceae } & \multirow[t]{4}{*}{ Aphelandra squarrosa Nees } & \multirow[t]{3}{*}{ S1 } & 13.03 .2009 \\
\hline & & & & 18.03 .2009 \\
\hline & & & & 26.05 .2010 \\
\hline & & & S3 & 28.04 .2010 \\
\hline & Araliaceae & Schefflera arboricola (Hayata) Merr. & GS & 05.03 .2010 \\
\hline & \multirow[t]{6}{*}{ Asteraceae } & \multirow[t]{2}{*}{ Chrysanthemum hybridum Guss. } & \multirow[t]{2}{*}{ S1 } & 17.02 .2009 \\
\hline & & & & 25.11 .2008 \\
\hline & & \multirow[t]{4}{*}{ Dendranthema sp. } & \multirow[t]{3}{*}{ S1 } & 13.02.2009 \\
\hline & & & & 13.03 .2009 \\
\hline & & & & 25.11 .2008 \\
\hline & & & S6 & 26.11 .2009 \\
\hline & \multirow[t]{12}{*}{ Malvaceae } & \multirow[t]{9}{*}{ Hibiscus rosa-sinensis $\mathrm{L}$. } & \multirow[t]{6}{*}{ GL } & 05.11 .2010 \\
\hline & & & & 08.10 .2010 \\
\hline & & & & 11.07 .2011 \\
\hline & & & & 18.09 .2009 \\
\hline & & & & 20.07 .2011 \\
\hline & & & & 31.08 .2010 \\
\hline & & & GV & 28.10 .2008 \\
\hline & & & \multirow[t]{2}{*}{$\mathrm{R} 1$} & 01.06 .2010 \\
\hline & & & & 19.07 .2010 \\
\hline & & \multirow[t]{3}{*}{ Hibiscus syriacus $\mathrm{L}$. } & GB & 04.08 .2010 \\
\hline & & & \multirow[t]{2}{*}{ GL } & 20.08 .2010 \\
\hline & & & & 30.07 .2010 \\
\hline & Primulaceae & Cyclamen persicum Mill. & S4 & 03.08 .2010 \\
\hline & Rosaceae & Eriobotrya japonica (Thunb.) Lindl. & R1 & 17.07.2009 \\
\hline & Scrophulariaceae & Hebe sp. & S1 & 01.07 .2010 \\
\hline
\end{tabular}




\begin{tabular}{|c|c|c|c|c|}
\hline Aphid species & Host plant family & Host plant species & $\begin{array}{l}\text { Green- } \\
\text { house }\end{array}$ & Date \\
\hline \multirow{4}{*}{$\begin{array}{l}\text { Aphis (Aphis) hederae Kaltenbach, } \\
1843\end{array}$} & \multirow[t]{4}{*}{ Araliaceae } & \multirow[t]{4}{*}{ Hedera helix L. } & GE & 23.07 .2010 \\
\hline & & & \multirow[t]{3}{*}{$\mathrm{S} 2$} & 06.08 .2010 \\
\hline & & & & 12.07 .2011 \\
\hline & & & & 27.05 .2010 \\
\hline \multirow{5}{*}{$\begin{array}{l}\text { Aphis (Aphis) nasturtii Kaltenbach, } \\
1843\end{array}$} & \multirow{4}{*}{ Malvaceae } & \multirow[t]{2}{*}{ Hibiscus rosa-sinensis $\mathrm{L}$. } & \multirow[t]{2}{*}{ GL } & 05.11 .2010 \\
\hline & & & & 31.08 .2010 \\
\hline & & \multirow[t]{2}{*}{ Hibiscus syriacus L. } & GL & 31.08 .2010 \\
\hline & & & $\mathrm{R} 1$ & 20.08 .2010 \\
\hline & Primulaceae & Cyclamen persicum Mill. & S4 & 03.08 .2010 \\
\hline \multirow{3}{*}{$\begin{array}{l}\text { Aphis (Aphis) nerii Boyer de } \\
\text { Fonscolombe, } 1841\end{array}$} & \multirow[t]{2}{*}{ Apocynaceae } & \multirow[t]{2}{*}{ Nerium oleander L. } & \multirow[t]{2}{*}{$\mathrm{R} 1$} & 17.07 .2009 \\
\hline & & & & 19.07 .2010 \\
\hline & Asclepiadaceae & Asclepias curassavica L. & $\mathrm{R} 1$ & 17.07 .2009 \\
\hline \multirow[t]{2}{*}{ Aphis (Aphis) sedi Kaltenbach, 1843} & \multirow[t]{2}{*}{ Crassulaceae } & Sedum adolphi Raym.-Hamet & GE & 27.05 .2009 \\
\hline & & Sedum glaucophyllum R. T. Clausen & GE & 27.05 .2009 \\
\hline \multirow{11}{*}{ Aphis (Aphis) spiraecola Patch, 1914} & \multirow[t]{2}{*}{ Aizoaceae } & \multirow[t]{2}{*}{ Aptenia cordifolia (L.f.) Schwantes } & \multirow[t]{2}{*}{ GL } & 14.07 .2010 \\
\hline & & & & 31.08 .2010 \\
\hline & Caprifoliaceae & Viburnum tinus L. & $\mathrm{R} 1$ & 01.06 .2010 \\
\hline & \multirow{2}{*}{ Malvaceae } & \multirow[t]{2}{*}{ Hibiscus rosa-sinensis $\mathrm{L}$. } & \multirow[t]{2}{*}{ GL } & 05.11 .2010 \\
\hline & & & & 08.10 .2010 \\
\hline & \multirow[t]{5}{*}{ Rosaceae } & \multirow[t]{2}{*}{ Aronia melanocarpa Nutt. ex Elliott } & \multirow[t]{2}{*}{ R1 } & 17.07.2009 \\
\hline & & & & 29.05 .2009 \\
\hline & & Spiraea douglasii Hook. & PV & 14.07 .2010 \\
\hline & & Spiraea japonica L. f. & PV & 14.07 .2010 \\
\hline & & Spiraea vanhouttei (Briot) Carrière & PV & 14.07 .2010 \\
\hline & Scrophulariaceae & Hebe sp. & $S 1$ & 01.07 .2010 \\
\hline $\begin{array}{l}\text { Aphis (Aphis) spiraephaga F. P. } \\
\text { Müller, } 1961\end{array}$ & Rosaceae & Spiraea douglasii Hook. & $\mathrm{S} 2$ & 06.08 .2010 \\
\hline $\begin{array}{l}\text { Aphis (Aphis) verbasci Schrank, } \\
1801\end{array}$ & Buddlejaceae & Buddleja davidii Franch. & GB & 04.08 .2010 \\
\hline Aphis (Aphis) sp. & Begoniaceae & Begonia semperflorens Hook. & PL & 19.08 .2010 \\
\hline Aulacorthum (Aulacorthum) solani & Acanthaceae & Aphelandra squarrosa Nees & $\mathrm{R} 2$ & 01.06 .2010 \\
\hline (Kaltenbach, 1843) & & & S1 & 28.04 .2010 \\
\hline & Aceraceae & Acer palmatum Thunb. & GB & 05.11 .2010 \\
\hline & Apocynaceae & $\begin{array}{l}\text { Mandevilla sanderii (Hemsl.) } \\
\text { Woodson }\end{array}$ & S5 & 12.07 .2011 \\
\hline & & Nerium oleander L. & GL & 08.06 .2011 \\
\hline & & Vinca major $\mathrm{L}$. & GL & 08.06 .2011 \\
\hline & & & & 28.03 .2011 \\
\hline & Araceae & Anthurium andraeanum Linden & GE & 23.07 .2010 \\
\hline & & & S1 & 28.04 .2010 \\
\hline & & Syngonium podophyllum Schott & GL & 08.06 .2011 \\
\hline & & Syngonium sp. & $\mathrm{R} 2$ & 01.06 .2010 \\
\hline & Araliaceae & Aralia japonica Thunb. & GL & 14.03 .2012 \\
\hline & & Hedera helix $\mathrm{L}$. & GL & 28.03 .2011 \\
\hline & & Schefflera arboricola (Hayata) Merr. & GL & 08.06 .2011 \\
\hline & & & $\mathrm{R} 1$ & 01.06 .2010 \\
\hline
\end{tabular}




\begin{tabular}{|c|c|c|c|c|}
\hline Aphid species & Host plant family & Host plant species & $\begin{array}{l}\text { Green- } \\
\text { house }\end{array}$ & Date \\
\hline \multirow{36}{*}{$\begin{array}{l}\text { Aulacorthum (Aulacorthum) solani } \\
\text { (Kaltenbach, 1843) }\end{array}$} & \multirow[t]{5}{*}{ Asteraceae } & Chrysanthemum hybridum Guss. & S4 & 12.07 .2011 \\
\hline & & $\begin{array}{l}\text { Dablia } \mathrm{x} \text { cultorum Thorsrud \& } \\
\text { Reisaeter }\end{array}$ & S5 & 26.05 .2010 \\
\hline & & Dendranthema sp. & $S 4$ & 12.07 .2011 \\
\hline & & Gerbera jamesonii Adlam & GL & 08.06.2011 \\
\hline & & Senecio macroglossus DC. & GV & 28.10 .2008 \\
\hline & Begoniaceae & Begonia elatior Hort. ex Steud. & S5 & 01.07 .2010 \\
\hline & Caesalpiniaceae & Gleditsia triacanthos $\mathrm{L}$. & GL & 08.06 .2011 \\
\hline & \multirow{2}{*}{ Caprifoliaceae } & Weigela floribunda C. A. Mey & R1 & 01.06 .2010 \\
\hline & & Weigela florida A. DC. & GL & 08.06 .2011 \\
\hline & \multirow[t]{13}{*}{ Geraniaceae } & \multirow[t]{4}{*}{ Pelargonium peltatum (L.) L'Hèr. } & \multirow[t]{3}{*}{ GL } & 05.11 .2010 \\
\hline & & & & 14.03 .2012 \\
\hline & & & & 28.03 .2011 \\
\hline & & & S2 & 19.05.2011 \\
\hline & & Pelargonium roseum Ehrh. & GL & 08.06.2011 \\
\hline & & \multirow[t]{8}{*}{ Pelargonium zonale (L.) L'Hèr. } & \multirow[t]{6}{*}{ GL } & 01.04 .2010 \\
\hline & & & & 05.11 .2010 \\
\hline & & & & 14.07 .2010 \\
\hline & & & & 18.09 .2009 \\
\hline & & & & 21.03 .2011 \\
\hline & & & & 27.04 .2010 \\
\hline & & & \multirow[t]{2}{*}{ S2 } & 19.05 .2011 \\
\hline & & & & 24.01 .2011 \\
\hline & \multirow[t]{3}{*}{ Lamiaceae } & Coleus $\mathrm{x}$ hybridus Hort. & GL & 08.06.2011 \\
\hline & & Mentha sp. & GB & 05.11 .2010 \\
\hline & & Thymus sp. & GB & 05.11 .2010 \\
\hline & Lauraceae & Persea americana Mill. & GL & 08.06 .2011 \\
\hline & Liliaceae & Tulipa sp. & S5 & 26.05 .2010 \\
\hline & \multirow[t]{5}{*}{ Malvaceae } & \multirow[t]{5}{*}{ Hibiscus rosa-sinensis $\mathrm{L}$. } & GB & 07.10 .2010 \\
\hline & & & \multirow[t]{4}{*}{ GL } & 08.06 .2011 \\
\hline & & & & 11.07 .2011 \\
\hline & & & & 28.03 .2011 \\
\hline & & & & 28.05 .2011 \\
\hline & Onagraceae & Fuchsia hybrida Hort. & GL & 08.06.2011 \\
\hline & \multirow[t]{2}{*}{ Solanaceae } & \multirow[t]{2}{*}{ Calibrachoa sp. } & \multirow[t]{2}{*}{ S5 } & 26.05 .2010 \\
\hline & & & & 28.04 .2010 \\
\hline & Verbenaceae & Verbena $\mathrm{x}$ hybrida Hort. ex Vilm. & S5 & 26.05 .2010 \\
\hline \multirow[t]{6}{*}{$\begin{array}{l}\text { Aulacorthum (Neomyzus) } \\
\text { circumflexum (Buckton, 1876) }\end{array}$} & \multirow[t]{2}{*}{ Acanthaceae } & Acanthus sp. & GB & $\begin{array}{l}\text { Tashev } \\
(1962)\end{array}$ \\
\hline & & Ruellia speciosa Lindau & GB & $\begin{array}{l}\text { Tashev } \\
(1962)\end{array}$ \\
\hline & Adiantaceae & Adianthum capillus-veneris $\mathrm{L}$. & GL & 21.03 .2011 \\
\hline & Amaryllidaceae & Nerine sp. & GB & $\begin{array}{l}\text { Tashev } \\
(1962)\end{array}$ \\
\hline & \multirow[t]{2}{*}{ Anthericaceae } & \multirow{2}{*}{$\begin{array}{l}\text { Chlorophytum comosum (Thunb.) } \\
\text { Jacques }\end{array}$} & \multirow[t]{2}{*}{ GL } & 17.02 .2005 \\
\hline & & & & 26.05 .2010 \\
\hline
\end{tabular}




\begin{tabular}{|c|c|c|c|c|}
\hline Aphid species & Host plant family & Host plant species & $\begin{array}{l}\text { Green- } \\
\text { house }\end{array}$ & Date \\
\hline \multirow{36}{*}{$\begin{array}{l}\text { Aulacorthum (Neomyzus) } \\
\text { circumflexum (Buckton, 1876) }\end{array}$} & \multirow[t]{4}{*}{ Apocynaceae } & Catharanthus sp. & GL & 28.03 .2011 \\
\hline & & \multirow[t]{3}{*}{ Vinca major $\mathrm{L}}$. & \multirow[t]{3}{*}{ GL } & 01.04 .2010 \\
\hline & & & & 08.06 .2011 \\
\hline & & & & 11.07.2011 \\
\hline & \multirow[t]{11}{*}{ Araceae } & \multirow[t]{2}{*}{ Alocasia macrorrhizos (L.) G. Don } & GB & $\begin{array}{l}\text { Tashev } \\
(1962)\end{array}$ \\
\hline & & & GL & 28.03 .2011 \\
\hline & & Anthurium andraeanum Linden & GE & 30.06 .2009 \\
\hline & & Calla sp. & GB & $\begin{array}{l}\text { Tashev } \\
(1962)\end{array}$ \\
\hline & & Colocasia antiquorum Schott & GB & $\begin{array}{l}\text { Tashev } \\
(1962)\end{array}$ \\
\hline & & \multirow[t]{5}{*}{ Syngonium podophyllum Schott } & \multirow[t]{5}{*}{ GL } & 01.04 .2010 \\
\hline & & & & 03.12 .2008 \\
\hline & & & & 17.02 .2009 \\
\hline & & & & 28.03 .2011 \\
\hline & & & & 30.06 .2009 \\
\hline & & Zantedeschia aethiopica (L.) Spreng. & GL & 08.06 .2011 \\
\hline & \multirow[t]{6}{*}{ Asteraceae } & Chrysanthemum hybridum Guss. & S5 & 28.04 .2010 \\
\hline & & Chrysanthemum indicum $\mathrm{L}$. & GB & $\begin{array}{l}\text { Tashev } \\
(1962)\end{array}$ \\
\hline & & Cineraria sp. & GB & $\begin{array}{l}\text { Tashev } \\
(1962)\end{array}$ \\
\hline & & Dendranthema sp. & S5 & 28.04 .2010 \\
\hline & & Gerbera jamesonii Adlam & GL & 28.03 .2011 \\
\hline & & Tagetes patula L. & GL & 21.03 .2011 \\
\hline & Begoniaceae & Begonia semperflorens Hook. & GL & 28.03 .2011 \\
\hline & \multirow[t]{2}{*}{ Bombacaceae } & \multirow[t]{2}{*}{ Ceiba pentandra Gaertn. } & \multirow[t]{2}{*}{ GL } & 21.03 .2011 \\
\hline & & & & 28.03 .2011 \\
\hline & Commelinaceae & Tradescantia sp. & GB & $\begin{array}{l}\text { Tashev } \\
(1962)\end{array}$ \\
\hline & Corylaceae & Carpinus betulus L. & GL & 08.06 .2011 \\
\hline & Ericaceae & Erica arborea $\mathrm{L}$. & GB & $\begin{array}{l}\text { Tashev } \\
(1962)\end{array}$ \\
\hline & \multirow[t]{2}{*}{ Hyacinthaceae } & Scilla maritima L. & GB & $\begin{array}{l}\text { Tashev } \\
(1962)\end{array}$ \\
\hline & & Scilla peruviana L. & GB & $\begin{array}{l}\text { Tashev } \\
(1962)\end{array}$ \\
\hline & Hydrocharitaceae & Hydrilla verticillata (L.f.) Royle & GB & $\begin{array}{l}\text { Tashev } \\
(1962)\end{array}$ \\
\hline & Iridaceae & Tritonia fenestrata Ker Gawl. & GB & $\begin{array}{l}\text { Tashev } \\
(1962)\end{array}$ \\
\hline & Lamiaceae & Coleus $\mathrm{x}$ hybridus Hort. & GB & $\begin{array}{l}\text { Tashev } \\
(1962)\end{array}$ \\
\hline & \multirow[t]{4}{*}{ Malvaceae } & \multirow[t]{2}{*}{ Abutilon hybridum Hort. } & GL & 08.06 .2011 \\
\hline & & & GB & $\begin{array}{l}\text { Tashev } \\
(1962)\end{array}$ \\
\hline & & \multirow[t]{2}{*}{ Hibiscus rosa-sinensis $\mathrm{L}$. } & \multirow[t]{2}{*}{ GL } & 03.12 .2008 \\
\hline & & & & 30.06 .2009 \\
\hline
\end{tabular}




\begin{tabular}{|c|c|c|c|c|}
\hline Aphid species & Host plant family & Host plant species & $\begin{array}{l}\text { Green- } \\
\text { house }\end{array}$ & Date \\
\hline \multirow[t]{16}{*}{$\begin{array}{l}\text { Aulacorthum (Neomyzus) } \\
\text { circumflexum (Buckton, 1876) }\end{array}$} & \multirow[t]{2}{*}{ Nymphaeaceae } & Nymphaea coerulea Lam. & GB & $\begin{array}{l}\text { Tashev } \\
(1962)\end{array}$ \\
\hline & & Nymphaea sp. & GB & $\begin{array}{l}\text { Tashev } \\
(1962)\end{array}$ \\
\hline & \multirow[t]{4}{*}{ Oxalidaceae } & Oxalis floribunda Lehm. & GB & $\begin{array}{l}\text { Tashev } \\
(1962)\end{array}$ \\
\hline & & \multirow[t]{2}{*}{ Oxalis sp. } & GL & 28.03.2011 \\
\hline & & & GB & $\begin{array}{l}\text { Tashev } \\
(1962)\end{array}$ \\
\hline & & Oxalis violacea $\mathrm{L}$. & GB & $\begin{array}{l}\text { Tashev } \\
(1962)\end{array}$ \\
\hline & Polypodiaceae & Polypodium vulgare $\mathrm{L}$. & GB & $\begin{array}{l}\text { Tashev } \\
(1962)\end{array}$ \\
\hline & \multirow[t]{3}{*}{ Primulaceae } & Cyclamen persicum Mill. & GL & 28.03 .2011 \\
\hline & & Cyclamen sp. & GB & $\begin{array}{l}\text { Tashev } \\
(1962)\end{array}$ \\
\hline & & Primula obconica Hance & GL & 28.03 .2011 \\
\hline & Ranunculaceae & Aquilegia vulgaris $\mathrm{L}$. & GL & 08.06 .2011 \\
\hline & Rosaceae & Aronia melanocarpa Nutt. ex Elliott & GL & 08.06.2011 \\
\hline & Rubiaceae & Hoffmannia refulgens Hemsl. & GE & 23.07.2010 \\
\hline & Salviniaceae & Salvinia auriculata Aubl. & GB & $\begin{array}{l}\text { Tashev } \\
(1962)\end{array}$ \\
\hline & Saxifragaceae & Saxifraga sarmentosa L.f. & GB & $\begin{array}{l}\text { Tashev } \\
(1962)\end{array}$ \\
\hline & Tiliaceae & $\begin{array}{l}\text { Sparmannia palmata Hort. ex } \\
\text { Lindl. }\end{array}$ & GB & $\begin{array}{l}\text { Tashev } \\
(1962)\end{array}$ \\
\hline \multirow{5}{*}{$\begin{array}{l}\text { Brachycaudus (Acaudus) cardui } \\
\text { (Linnaeus, 1758) }\end{array}$} & \multirow[t]{5}{*}{ Asteraceae } & Chrysanthemum frutescens $\mathrm{L}$. & GB & 05.11 .2010 \\
\hline & & $\begin{array}{l}\text { Dablia } \mathrm{x} \text { cultorum Thorsrud \& } \\
\text { Reisaeter }\end{array}$ & SM & 05.09 .2011 \\
\hline & & \multirow[t]{3}{*}{ Senecio cineraria DC. } & \multirow[t]{3}{*}{ GL } & 08.06.2011 \\
\hline & & & & 11.07.2011 \\
\hline & & & & 28.03 .2011 \\
\hline $\begin{array}{l}\text { Brachycaudus (Brachycaudus) } \\
\text { helichrysi (Kaltenbach, 1843) }\end{array}$ & Asteraceae & Senecio mikanioides Otto ex Walp. & GB & 04.08 .2010 \\
\hline $\begin{array}{l}\text { Cinara (Cinara) neubergi (Arnhart, } \\
\text { 1930) }\end{array}$ & Pinaceae & Pinus pinaster Aiton & R1 & 29.05 .2009 \\
\hline \multirow[t]{12}{*}{ Idiopterus nephrelepidis Davis, 1909} & Adiantaceae & Adianthum capillus-veneris $\mathrm{L}$. & S1 & 25.05 .2011 \\
\hline & Araceae & Syngonium podophyllum Schott & GL & 23.03 .2011 \\
\hline & Aspleniaceae & Asplenium nidus $\mathrm{L}$. & V1 & 13.07.2009 \\
\hline & Blechnaceae & Blechnum sp. & S1 & 25.05 .2011 \\
\hline & \multirow[t]{6}{*}{ Davalliaceae } & \multirow[t]{6}{*}{ Nephrolepis exaltata (L.) Schott } & \multirow[t]{2}{*}{ GL } & 25.04 .2012 \\
\hline & & & & 30.03 .2012 \\
\hline & & & \multirow[t]{2}{*}{ S1 } & 13.03 .2009 \\
\hline & & & & 25.05 .2011 \\
\hline & & & \multirow[t]{2}{*}{ S3 } & 06.08 .2010 \\
\hline & & & & 19.05 .2011 \\
\hline & Piperaceae & Peperomia clusiifolia Hook. & GL & 28.03 .2011 \\
\hline & Polypodiaceae & $\begin{array}{l}\text { Platycerium bifurcatum (Cav.) C. } \\
\text { Chr. }\end{array}$ & V1 & 13.07.2009 \\
\hline
\end{tabular}




\begin{tabular}{|c|c|c|c|c|}
\hline Aphid species & Host plant family & Host plant species & $\begin{array}{l}\text { Green- } \\
\text { house }\end{array}$ & Date \\
\hline \multirow[t]{2}{*}{ Idiopterus nephrelepidis Davis, 1909} & \multirow[t]{2}{*}{ Pteridaceae } & Pteris cretica $\mathrm{L}$. & GL & 28.03 .2011 \\
\hline & & Pteris sp. & S1 & 25.05 .2011 \\
\hline \multirow{21}{*}{$\begin{array}{l}\text { Macrosiphoniella (Macrosiphoniella) } \\
\text { sanborni (Gillette, 1908) }\end{array}$} & \multirow[t]{21}{*}{ Asteraceae } & \multirow[t]{8}{*}{ Chrysanthemum hybridum Guss. } & S1 & 20.10 .2010 \\
\hline & & & S4 & 12.07 .2011 \\
\hline & & & \multirow[t]{4}{*}{ S5 } & 07.10 .2010 \\
\hline & & & & 18.12 .2008 \\
\hline & & & & 26.05 .2010 \\
\hline & & & & 28.04 .2010 \\
\hline & & & \multirow[t]{2}{*}{ S6 } & 01.11 .2011 \\
\hline & & & & 26.11 .2009 \\
\hline & & Chrysanthemum indicum L. & GB & $\begin{array}{l}\text { Tashev } \\
(1962)\end{array}$ \\
\hline & & \multirow[t]{12}{*}{ Dendranthema sp. } & GE & 27.05 .2009 \\
\hline & & & GV & 28.10 .2008 \\
\hline & & & \multirow[t]{2}{*}{$\mathrm{R} 1$} & 23.07 .2010 \\
\hline & & & & 29.05 .2009 \\
\hline & & & S1 & 20.10 .2010 \\
\hline & & & S4 & 12.07 .2011 \\
\hline & & & \multirow[t]{4}{*}{ S5 } & 07.10 .2010 \\
\hline & & & & 18.12 .2008 \\
\hline & & & & 26.05 .2010 \\
\hline & & & & 28.04 .2010 \\
\hline & & & \multirow[t]{2}{*}{ S6 } & 01.11 .2011 \\
\hline & & & & 26.11 .2009 \\
\hline \multirow{10}{*}{$\begin{array}{l}\text { Macrosiphum (Macrosiphum) } \\
\text { euphorbiae (Thomas, 1878) }\end{array}$} & Acanthaceae & Aphelandra squarrosa Nees & $\mathrm{R} 2$ & 01.06 .2010 \\
\hline & Anthericaceae & $\begin{array}{l}\text { Chlorophytum comosum (Thunb.) } \\
\text { Jacques }\end{array}$ & $\mathrm{R} 2$ & 01.06 .2010 \\
\hline & \multirow[t]{2}{*}{ Apocynaceae } & $\begin{array}{l}\text { Mandevilla sanderii (Hemsl.) } \\
\text { Woodson }\end{array}$ & $\mathrm{R} 2$ & 01.06 .2010 \\
\hline & & Vinca major L. & $\mathrm{R} 1$ & 29.05 .2009 \\
\hline & Araliaceae & Schefflera arboricola (Hayata) Merr. & R1 & 01.06 .2010 \\
\hline & Asteraceae & Cineraria sp. & GB & $\begin{array}{l}\text { Tashev } \\
(1962)\end{array}$ \\
\hline & Hydrangeaceae & Hydrangea hortensis Sm. & GB & $\begin{array}{l}\text { Tashev } \\
(1962)\end{array}$ \\
\hline & \multirow{3}{*}{ Malvaceae } & \multirow[t]{3}{*}{ Hibiscus rosa-sinensis $\mathrm{L}$. } & \multirow[t]{2}{*}{ GL } & 28.03 .2011 \\
\hline & & & & 30.06 .2009 \\
\hline & & & $\mathrm{R} 2$ & 01.06 .2010 \\
\hline \multirow{2}{*}{$\begin{array}{l}\text { Macrosiphum (Macrosiphum) rosae } \\
\text { (Linnaeus, 1758) }\end{array}$} & \multirow[t]{2}{*}{ Rosaceae } & Rosa hybrida Vill. & GE & 27.05 .2009 \\
\hline & & Rosa rugosa Thunb. & GB & 05.11 .2010 \\
\hline \multirow[t]{5}{*}{ Myzus (Myzus) ornatus Laing, 1932} & \multirow[t]{3}{*}{ Acanthaceae } & Acanthus sp. & GB & $\begin{array}{l}\text { Tashev } \\
(1962)\end{array}$ \\
\hline & & Fittonia argyroneura E. Coem. & GB & $\begin{array}{l}\text { Tashev } \\
(1962)\end{array}$ \\
\hline & & Ruellia speciosa Lindau & GB & $\begin{array}{l}\text { Tashev } \\
(1962)\end{array}$ \\
\hline & Amaranthaceae & Iresine herbstii Hook. & GV & 25.05 .2009 \\
\hline & Araliaceae & Aralia sieboldii Hort. ex K. Koch & GB & $\begin{array}{l}\text { Tashev } \\
(1962)\end{array}$ \\
\hline
\end{tabular}




\begin{tabular}{|c|c|c|c|c|}
\hline Aphid species & Host plant family & Host plant species & $\begin{array}{l}\text { Green- } \\
\text { house }\end{array}$ & Date \\
\hline \multirow[t]{22}{*}{ Myzus (Myzus) ornatus Laing, 1932} & Asparagaceae & Asparagus sp. & GB & $\begin{array}{l}\text { Tashev } \\
(1962)\end{array}$ \\
\hline & \multirow[t]{3}{*}{ Asteraceae } & $\begin{array}{l}\text { Centaurea macrocephala Muss. } \\
\text { Pushk. ex Willd. }\end{array}$ & GB & $\begin{array}{l}\text { Tashev } \\
(1962)\end{array}$ \\
\hline & & Chrysanthemum indicum $\mathrm{L}$. & GB & $\begin{array}{l}\text { Tashev } \\
(1962)\end{array}$ \\
\hline & & Cineraria sp. & GB & $\begin{array}{l}\text { Tashev } \\
(1962)\end{array}$ \\
\hline & Begoniaceae & Begonia sp. & GB & $\begin{array}{l}\text { Tashev } \\
(1962)\end{array}$ \\
\hline & Brassicaceae & Arabis alpina $\mathrm{L}$. & GB & $\begin{array}{l}\text { Tashev } \\
(1962)\end{array}$ \\
\hline & \multirow[t]{3}{*}{ Ericaceae } & Erica australis $\mathrm{L}$. & GB & $\begin{array}{l}\text { Tashev } \\
(1962)\end{array}$ \\
\hline & & Erica lusitanica Rudolphi & GB & $\begin{array}{l}\text { Tashev } \\
(1962)\end{array}$ \\
\hline & & Erica sp. & GB & $\begin{array}{l}\text { Tashev } \\
(1962)\end{array}$ \\
\hline & Fabaceae & Lupinus sp. & GB & $\begin{array}{l}\text { Tashev } \\
(1962)\end{array}$ \\
\hline & Gesneriaceae & Saintpaulia ionantha $\mathrm{H}$. Wendl. & GB & $\begin{array}{l}\text { Tashev } \\
(1962)\end{array}$ \\
\hline & Hydrangeaceae & Hydrangea hortensis $\mathrm{Sm}$. & GB & $\begin{array}{l}\text { Tashev } \\
(1962)\end{array}$ \\
\hline & \multirow[t]{2}{*}{ Lamiaceae } & \multirow[t]{2}{*}{ Coleus $\mathrm{x}$ hybridus Hort. } & GV & 25.05 .2009 \\
\hline & & & GB & $\begin{array}{l}\text { Tashev } \\
(1962)\end{array}$ \\
\hline & Mimosaceae & Acacia floribunda Willd. & GB & $\begin{array}{l}\text { Tashev } \\
(1962)\end{array}$ \\
\hline & Oxalidaceae & Oxalis floribunda Lehm. & GB & $\begin{array}{l}\text { Tashev } \\
(1962)\end{array}$ \\
\hline & Primulaceae & Primula sp. & GB & $\begin{array}{l}\text { Tashev } \\
(1962)\end{array}$ \\
\hline & Saxifragaceae & Heuchera sp. & GB & $\begin{array}{l}\text { Tashev } \\
(1962)\end{array}$ \\
\hline & Scrophulariaceae & Digitalis purpurea $\mathrm{L}$. & GB & $\begin{array}{l}\text { Tashev } \\
(1962)\end{array}$ \\
\hline & Urticaceae & Laportea gigas Wedd. & GB & $\begin{array}{l}\text { Tashev } \\
(1962)\end{array}$ \\
\hline & Valerianaceae & Valeriana montana $\mathrm{L}$. & GB & $\begin{array}{l}\text { Tashev } \\
(1962)\end{array}$ \\
\hline & Violaceae & Viola sp. & GB & $\begin{array}{l}\text { Tashev } \\
(1962)\end{array}$ \\
\hline \multirow[t]{4}{*}{$\begin{array}{l}\text { Myzus (Nectarosiphon) ascalonicus } \\
\text { Doncaster, } 1946\end{array}$} & Cucurbitaceae & Lagenaria vulgaris Ser. & GB & $\begin{array}{l}\text { Tashev } \\
(1962)\end{array}$ \\
\hline & Geraniaceae & Pelargonium sp. & GB & $\begin{array}{l}\text { Tashev } \\
(1962)\end{array}$ \\
\hline & Hydrangeaceae & Hydrangea hortensis $\mathrm{Sm}$. & GB & $\begin{array}{l}\text { Tashev } \\
(1962)\end{array}$ \\
\hline & Lamiaceae & Salvia sp. & GB & $\begin{array}{l}\text { Tashev } \\
(1962)\end{array}$ \\
\hline
\end{tabular}




\begin{tabular}{|c|c|c|c|c|}
\hline Aphid species & Host plant family & Host plant species & $\begin{array}{l}\text { Green- } \\
\text { house }\end{array}$ & Date \\
\hline \multirow{3}{*}{$\begin{array}{l}\text { Myzus (Nectarosiphon) ascalonicus } \\
\text { Doncaster, } 1946\end{array}$} & Malvaceae & Hibiscus rosa-sinensis $\mathrm{L}$. & GL & 28.03 .2011 \\
\hline & Resedaceae & Reseda odorata L. & GB & $\begin{array}{l}\text { Tashev } \\
(1962)\end{array}$ \\
\hline & Scrophulariaceae & Hebe sp. & S1 & 01.07 .2010 \\
\hline \multirow{37}{*}{$\begin{array}{l}\text { Myzus (Nectarosiphon) persicae } \\
\text { (Sulzer, 1776) }\end{array}$} & \multirow[t]{3}{*}{ Acanthaceae } & Aphelandra aurantiaca Lindl. & GB & 07.10 .2010 \\
\hline & & \multirow[t]{2}{*}{ Thunbergia coccinea Wall. } & \multirow[t]{2}{*}{ GB } & 09.03 .2009 \\
\hline & & & & 20.12 .2008 \\
\hline & Agavaceae & Cordyline terminalis Kunth. & GS & 05.03 .2010 \\
\hline & Aizoaceae & Aptenia cordifolia (L.f.) Schwantes & GL & 24.01 .2011 \\
\hline & Amaranthaceae & Pleuropetalum darwinii Hook. f. & GS & 17.03 .2009 \\
\hline & \multirow[t]{2}{*}{ Anthericaceae } & \multirow{2}{*}{$\begin{array}{l}\text { Chlorophytum comosum (Thunb.) } \\
\text { Jacques }\end{array}$} & GK & 31.05 .2010 \\
\hline & & & GL & 11.07.2011 \\
\hline & \multirow[t]{4}{*}{ Apocynaceae } & Catharanthus sp. & GL & 28.03 .2011 \\
\hline & & \multirow{3}{*}{$\begin{array}{l}\text { Mandevilla sanderii (Hemsl.) } \\
\text { Woodson }\end{array}$} & \multirow[t]{3}{*}{ S5 } & 01.07 .2010 \\
\hline & & & & 03.08 .2010 \\
\hline & & & & 26.05 .2010 \\
\hline & \multirow[t]{3}{*}{ Araceae } & Anthurium andraeanum Linden & $\mathrm{V} 2$ & 14.07.2009 \\
\hline & & Syngonium podophyllum Schott & GL & 24.01 .2011 \\
\hline & & Zantedeschia aethiopica (L.) Spreng. & GV & 28.10 .2008 \\
\hline & \multirow[t]{5}{*}{ Araliaceae } & Hedera helix $\mathrm{L}$. & GL & 14.03 .2012 \\
\hline & & \multirow[t]{4}{*}{ Schefflera arboricola (Hayata) Merr. } & \multirow[t]{3}{*}{ GB } & 02.09 .2010 \\
\hline & & & & 04.08 .2010 \\
\hline & & & & 07.10 .2010 \\
\hline & & & $\mathrm{R} 1$ & 01.06 .2010 \\
\hline & \multirow[t]{9}{*}{ Asteraceae } & Bellis perennis L. & GB & 20.10 .2010 \\
\hline & & Chrysanthemum hybridum Guss. & S1 & 13.03 .2009 \\
\hline & & Chrysanthemum indicum $\mathrm{L}$. & GB & $\begin{array}{l}\text { Tashev } \\
(1962)\end{array}$ \\
\hline & & Cineraria sp. & GB & $\begin{array}{l}\text { Tashev } \\
(1962)\end{array}$ \\
\hline & & Dendranthema sp. & S1 & 13.03 .2009 \\
\hline & & $\begin{array}{l}\text { Gazania heterophylla Willd. ex } \\
\text { Steud. }\end{array}$ & S4 & 26.05 .2010 \\
\hline & & Senecio hybridus Scheidw. & S1 & 13.03 .2009 \\
\hline & & Senecio rowleyanus $\mathrm{H}$. Jacobsen & GB & 18.12 .2008 \\
\hline & & Zinnia elegans Jacq. & GE & 01.06 .2010 \\
\hline & Bignoniaceae & Campsis radicans (L.) Seem. & S5 & 30.08 .2010 \\
\hline & Bombacaceae & Ceiba pentandra Gaertn. & GL & 14.03 .2012 \\
\hline & \multirow[t]{2}{*}{ Brassicaceae } & \multirow[t]{2}{*}{ Arabis alpina L. } & GB & 20.10 .2010 \\
\hline & & & GL & 24.01 .2011 \\
\hline & Cactaceae & Zygocactus truncatus K.Schum. & GS & 05.03 .2010 \\
\hline & \multirow[t]{2}{*}{ Caprifoliaceae } & \multirow[t]{2}{*}{ Viburnum tinus L. } & \multirow[t]{2}{*}{ GB } & 02.09 .2010 \\
\hline & & & & 07.10 .2010 \\
\hline & Caryophyllaceae & $\begin{array}{l}\text { Dianthus hybridus Schmidt ex } \\
\text { Tausch }\end{array}$ & S6 & 31.05 .2011 \\
\hline
\end{tabular}




\begin{tabular}{|c|c|c|c|c|}
\hline Aphid species & Host plant family & Host plant species & $\begin{array}{l}\text { Green- } \\
\text { house }\end{array}$ & Date \\
\hline \multirow{27}{*}{$\begin{array}{l}\text { Myzus (Nectarosiphon) persicae } \\
\text { (Sulzer, 1776) }\end{array}$} & \multirow[t]{4}{*}{ Convolvulaceae } & \multirow{3}{*}{$\begin{array}{l}\text { Dichondra repens J. R. Forst \& G. } \\
\text { Forst }\end{array}$} & \multirow[t]{3}{*}{ S4 } & 01.07 .2010 \\
\hline & & & & 26.05 .2010 \\
\hline & & & & 28.04 .2010 \\
\hline & & Ipomoea purpurea (L.) Roth & S4 & 28.04 .2010 \\
\hline & Droseraceae & Dionea sp. & S3 & 06.08 .2010 \\
\hline & Gesneriaceae & Aeschynanthus radicans Jack & GB & 05.03 .2010 \\
\hline & Hydrangeaceae & Hydrangea hortensis Sm. & GB & $\begin{array}{l}\text { Tashev } \\
(1962)\end{array}$ \\
\hline & Lamiaceae & Thymus sp. & GB & 05.11 .2010 \\
\hline & \multirow[t]{6}{*}{ Malvaceae } & \multirow[t]{4}{*}{ Hibiscus rosa-sinensis $\mathrm{L}$. } & \multirow{2}{*}{ GB } & 07.10 .2010 \\
\hline & & & & 09.03 .2009 \\
\hline & & & GL & 24.01 .2011 \\
\hline & & & S6 & 04.11 .2009 \\
\hline & & Hibiscus sp. & GS & 17.12 .2008 \\
\hline & & Hibiscus syriacus $\mathrm{L}$. & GL & 28.05 .2011 \\
\hline & \multirow[t]{3}{*}{ Nyctaginaceae } & \multirow[t]{3}{*}{ Bougainvillea glabra Choisy } & GB & 04.08 .2010 \\
\hline & & & R1 & 01.06 .2010 \\
\hline & & & S4 & 28.04 .2010 \\
\hline & Oleaceae & Jasminum officinale $\mathrm{L}$. & GB & 09.03 .2009 \\
\hline & Primulaceae & Cyclamen persicum Mill. & S1 & 13.03.2009 \\
\hline & \multirow[t]{7}{*}{ Solanaceae } & Calibrachoa sp. & S5 & 26.05 .2010 \\
\hline & & Datura hybrida Ten. & GE & 27.05 .2009 \\
\hline & & \multirow{3}{*}{$\begin{array}{l}\text { Solandra maxima (Sessè \& Moc.) } \\
\text { P. S. Green }\end{array}$} & \multirow[t]{3}{*}{ GB } & 04.08 .2010 \\
\hline & & & & 05.03 .2010 \\
\hline & & & & 07.10 .2010 \\
\hline & & Solandra sp. & GB & 26.05 .2010 \\
\hline & & Solanum sp. & GB & 26.05 .2010 \\
\hline & Zamiaceae & Zamia pumila L. & GB & 18.12 .2008 \\
\hline $\begin{array}{l}\text { Ovatus (Ovatus) crataegarius } \\
\text { (Walker, 1850) }\end{array}$ & Lamiaceae & Monarda didyma $\mathrm{L}$. & R1 & 01.06 .2010 \\
\hline $\begin{array}{l}\text { Periphyllus californiensis (Shinji, } \\
\text { 1917) }\end{array}$ & Aceraceae & Acer palmatum Thunb. & GB & 20.10 .2010 \\
\hline $\begin{array}{l}\text { Prociphilus (Meliarhizophagus) } \\
\text { fraxinifolii (Riley in Riley \& } \\
\text { Monell, 1879) }\end{array}$ & Oleaceae & Fraxinus sp. & R1 & 01.06 .2010 \\
\hline Prociphilus sp. & Oleaceae & Fraxinus excelsior $\mathrm{L}$. & GL & 17.02. 2009 \\
\hline $\begin{array}{l}\text { Rhopalosiphum nymphaeae } \\
\text { (Linnaeus, 1761) }\end{array}$ & Nymphaeaceae & Nymphaea alba L. & GV & 28.10 .2008 \\
\hline \multirow{3}{*}{$\begin{array}{l}\text { Rhopalosiphum padi (Linnaeus, } \\
\text { 1758) }\end{array}$} & Asphodelaceae & Kniphophia uvaria (L.) Hook. & R1 & 29.05 .2009 \\
\hline & Poaceae & Agrostis stolonifera L. & S4 & 18.11 .2010 \\
\hline & & $\begin{array}{l}\text { Festuca ovina L. subsp. glauca } \\
\text { (Vill.) O. Bolòs \& Vigo }\end{array}$ & R1 & 29.05 .2009 \\
\hline \multirow{2}{*}{$\begin{array}{l}\text { Tinocallis (Sarucallis) } \\
\text { kahawaluokalani (Kirkaldy, 1907) }\end{array}$} & \multirow[t]{2}{*}{ Lythraceae } & \multirow[t]{2}{*}{ Lagerstroemia indica $\mathrm{L}$. } & \multirow[t]{2}{*}{ R1 } & 01.06 .2010 \\
\hline & & & & 29.05 .2009 \\
\hline
\end{tabular}

* The specimens of Aphis (Aphis) fabae are not identified to the subspecies level. 
(3 greenhouses, 8 samples, $2.9 \%$ ). All other species were found very rarely and were represented by 5 or less samples $(<1.8 \%)$.

The present study indicates that in greenhouses with a permanent plant composition, aphid infestations are more frequent, more widespread, and are caused by a greater variety of species compared to infestations in greenhouses with a constant circulation of plant species (Fig. 2).

The widest host range was shown by Myzus persicae (43 hosts, 38 \%), Aulacorthum solani (32 hosts, $28 \%$ ), Aulacorthum circumflexum (23 hosts, 20 \%), Aphis gossypii (9 hosts, 8 \%), Aphis spiraecola (8 hosts, $7 \%$ ), Aphis fabae (7 hosts, $6 \%$ ) and Macrosiphum euphorbiae (6 hosts, $5 \%$ ).

Periphyllus californiensis and Aphis (Aphis) fabae mordvilkoi are reported for the first time for Bulgaria. Furthermore, Aphis spiraecola has been found in new localities and has widened its host range in this country. So far, this species had been reported only on apple by Rasheva and Andreev (2007). All hosts reported in the present study for Aphis spiraecola are new for Bulgaria.

The list of host plants includes 114 species from 95 genera and 58 families. The most frequently infested plant species belong to Asteraceae (50 samples) and Malvaceae (37 samples). Eighteen samples were collected from Araceae; 16 samples from Apocynaceae; 15 samples from Araliaceae; 13 samples from Geraniaceae; and 10 samples from Acanthaceae, Solanaceae and Rosaceae.

The most frequently infested plants belong to the genera Hibiscus (12.9 \%, 36 samples), Dendranthema (6.8 \%, 19 samples), Chrysanthemum (5\%, 14 samples) and Pelargonium (4.7\%, 13 samples).

The highest diversity of aphid species was observed on Hibiscus rosa-sinensis and consists of 9 species (from 29 samples). Five species were identified on Dendranthema sp. (19 samples), Chrysanthemum hybridum (13 samples) and Anthurium andreanum

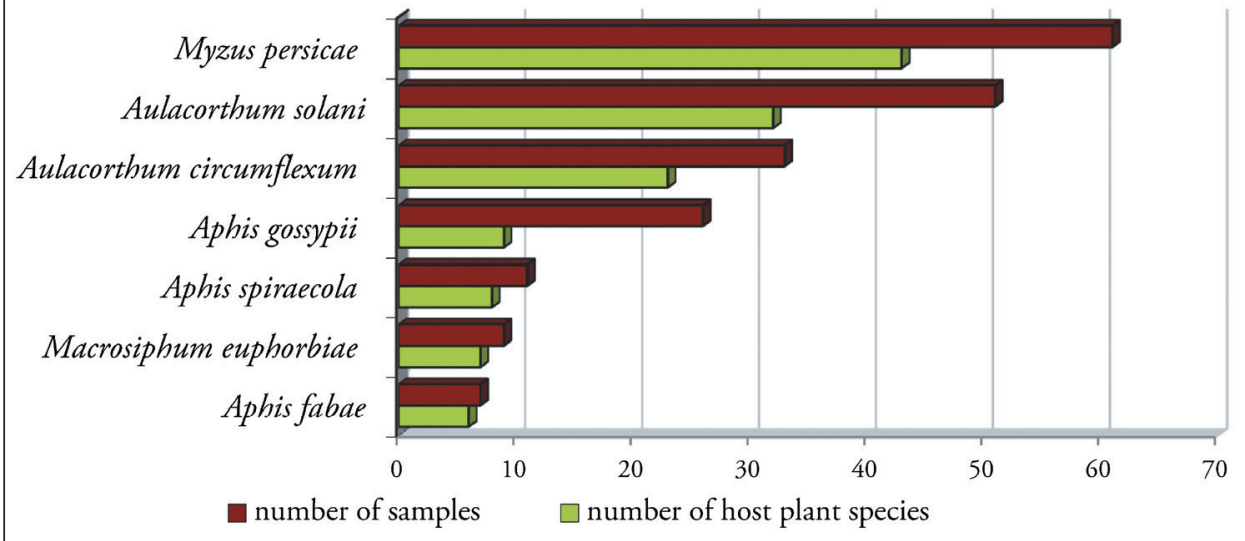

Figure I. Number of samples and number of host plant species found by polyphagous aphid species. 


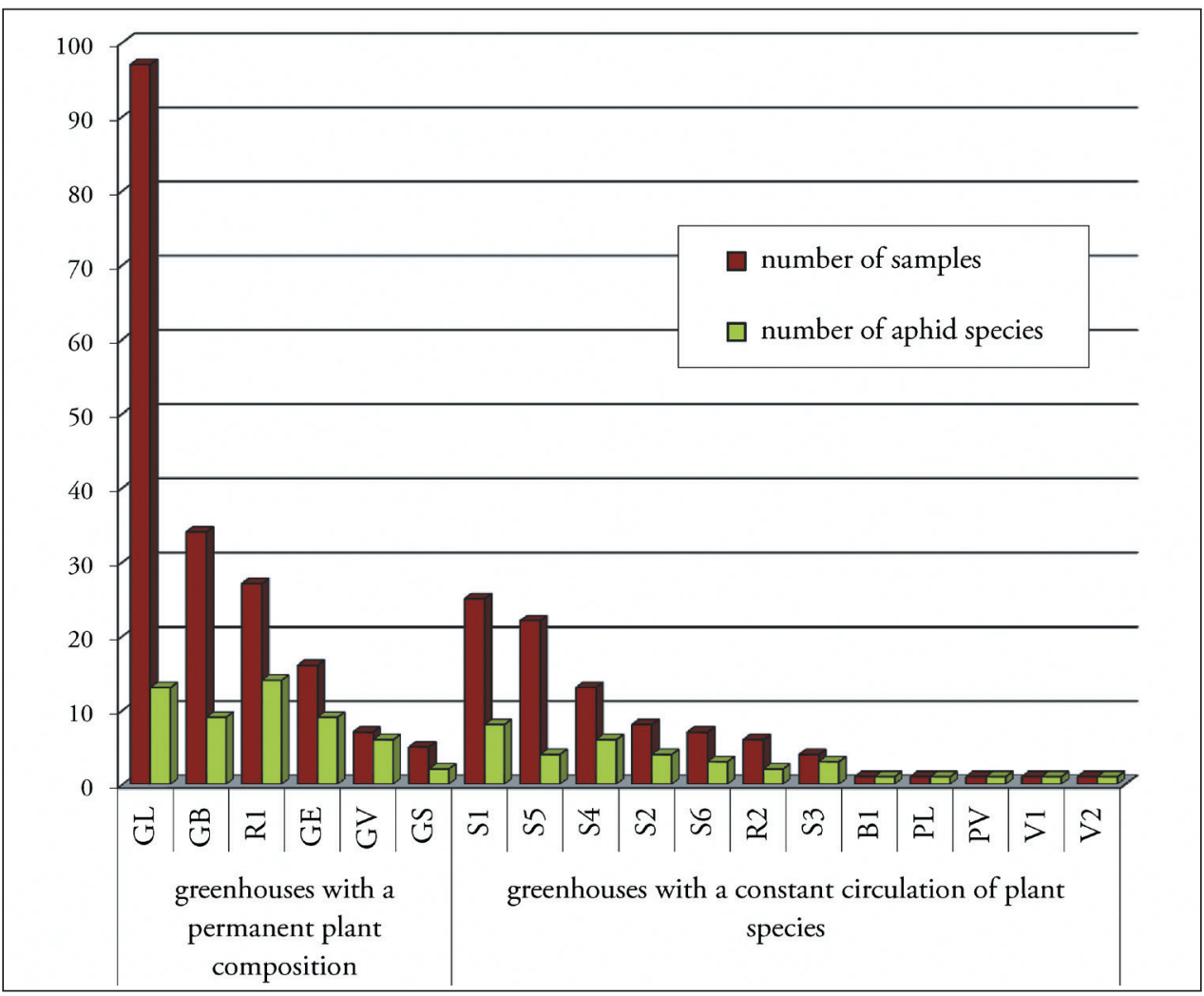

Figure 2. Distribution of number of samples and number of aphid species by greenhouses.

(6 samples). Four species were found on Cyclamen persicum, Shefflera arboricola and Syngonium podophyllum. Three species were recorded on Aptenia cordifolia, Bougainvillea glabra, Chlorophytum comosum, Hebe sp., Hedera helix, Hibiscus syriacus, Mandevilla sanderi and Vinca major.

\section{Conclusion}

The aphids established on ornamental plants in greenhouses in Bulgaria comprise 33 species and one subspecies from 13 genera and 5 subfamilies. The most widespread aphid species is Myzus persicae, followed by Aulacorthum solani and Aphis gossypii. The widest host ranges were shown by Myzus persicae, Aulacorthum solani and Aulacorthum circumflexum.

The list of host plants includes 114 species from 95 genera and 58 families. The most infested plants belong to the genera Hibiscus and Dendranthema. The largest number of aphid species was detected on Hibiscus (9 species). 


\section{Acknowledgement}

This study constitutes a part of a project funded in the frame of the state budget resources of the University of Forestry (Sofia, Bulgaria). Additional support is given by the Ministry of Sciences and Environment Protection of Serbia (Grant 43001).

\section{References}

Achremowicz J, Maślanka L, Obrocka E (1986) Z badań nad fauną mszyc uszkadzających szklarniowe i doniczkowe roślin ozdobne. Zeszyty Problemówe Postępów Nauk Rolniczych 329: $57-68$.

Blackman RL, Eastop VF (1994) Aphids on the World's Trees. An Identification and Information Guide. CAB International in association with The Natural History Museum, 1016 pp.

Blackman RL, Eastop VF (2000) Aphids on the World's Crops. An Identification and Information Guide. Second Edition. The Natural History Museum, London, 466 pp.

Blackman RL, Eastop VF (2006) Aphids on the World's Herbaceous Plants and Shrubs. Department of Entomology, The Natural History Museum, London, 1439 pp.

Cichocka E (1992) Glasshouse aphids in Poland. Aphids and Other Homopterous Insects 3: $13-32$.

Cichocka E, Goszczynski W (1975) Mszyce (Homoptera, Aphidoidea) szkodniki roślin uprawianych pod szkłem. Fragmenta Faunistica 20 (17): 273-305.

Hille Ris Lambers D (1950) On mounting aphids and other soft-skinned insects. Entomologische Berichten 13: 55-58.

Łabanowski G (2008) Aphids (Hemiptera, Aphidoidea) on ornamental plants under cover. Aphids and Other Hemipterous Insects 14: 21-37.

Rafi U, Usmani MK, Akhta MS (2010) Aphids of ornamental plants and winter vegetables and their aphidiine parasitoids (Hymenoptera: Braconidae) in Aligarh region, Uttar Pradesh. Journal of Threatened Taxa 2 (9): 1162-1164.

Rasheva D, Andreev R (2007) Aphis spiraecola Patch. (Hemiptera: Aphididae) - a new pest on apple in Bulgaria. Acta entomologica bulgarica 13 (1/2): 91-97. [In Bulgarian with English abstract]

Shaposhnikov GCh (1964) Suborder Aphidinea. In: Bey-Bienko GYa (Ed) Keys to the Insects of the European Part of the USSR. I (Opredelitel nasekomykh evropeiskoi chasti SSSR. I). Nauka, Moscow-Leningrad, 489-616. [In Russian]

Stroyan HLG (1984) Handbook for the Identification of British Insects. Vol. 2. Part 6: AphidsPterocommatinae and Aphidinae (Aphidini). Royal Entomological Society of London, London, $232 \mathrm{pp}$.

Tashev DG (1962) Beobachtungen über Blattläuse (Hom., Aphid.) an Treibhauspflanzen in Bulgarien. Annuaire de l'Université de Sofia, Faculté de biologie, géologie et géographie, Biologie (Zoologie) 54-55 (1): 171-191. [In Bulgarian with German summary]

Weiss HB (1916) The insect fauna of New Jersey greenhouses exclusive of the Coccidae. Journal of the New York Entomological Society 24 (2): 144-150. 